\title{
Development and evaluation of 12-lead electrocardiogram in the left free wall of accessory pathway localization in patients with typical Wolff-Parkinson-White syndrome
}

\author{
Si Dung Chu ${ }^{1,2}, *$, Khanh Quoc Pham ${ }^{1,2}$, Dong Van Tran²
}

${ }^{1}$ School of Medicine and Pharmacy, Vietnam National University, Hanoi, Viet Nam

${ }^{2}$ Vietnam National Heart Institute, Bachmai Hospital, Hanoi, Viet Nam

\section{Correspondence}

Si Dung Chu, School of Medicine and Pharmacy, Vietnam National University, Hanoi, Viet Nam

Vietnam National Heart Institute, Bachmai Hospital, Hanoi, Viet Nam

Email: chudungsi@gmail.com

History

- Received: 15 August 2018

- Accepted: 07 October 2018

- Published: 30 November 2018

DOI :

https://doi.org/10.15419/bmrat.v5i11.502

\section{Check for updates}

\section{Copyright}

(๑) Biomepress. This is an openaccess article distributed under the terms of the Creative Commons Attribution 4.0 International license.

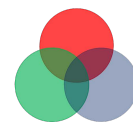

BioMedPress The Open Access Publisher

\begin{abstract}
Objectives: This study was designed to characterize 12-lead electrocardiogram (ECG) for localization of the left free wall lateral accessory pathway (AP) in patients with typical Wolff-Parkinson-White (WPW) syndrome, to develop a new algorithm ECG for localizing APs, and to test the accuracy of the algorithm prospectively. Method: We studied 129 patients; 84 patients had typical WPW syndrome with single anterograde AP identified by successful radiofrequency catheter ablation (RFCA), and were enrolled to build a new ECG algorithm for localizing left free wall APs. Then, the algorithm was tested prospectively in 45 patients and compared with the location of APs successfully ablated by RFCA. Results: We found that the 12-lead ECG parameters in typical WPW syndrome, such as delta wave polarity in V1, R/S ratio in V1, transition of the QRS complex, and delta wave polarity in inferior, lead to diagnosis and localization of APs, with highest accuracy predicted from $74.5 \%-100 \%$, and for development of a new ECG algorithm. From the 45 patients who were prospectively evaluated by the newly derived algorithm for the left free wall pathways, the sensitivity and specificity was high (from 75-100\%). Conclusion: The 12-lead ECG parameters in typical WPW syndrome are closely related to left free wall AP localization and can be used to develop a new ECG algorithm by the parameters above. Moreover, the new ECG algorithm can predict the location of APs with high accuracy.

Key words: 12-lead ECG, Accessory pathway localization, Left free wall, WPW syndrome
\end{abstract}

\section{INTRODUCTION}

Wolff-Parkinson-White syndrome (WPW) is a form of ventricular preexcitation in which part of the ventricular myocardium is depolarized early by one or more accessory pathways (APs) that bypass the atrioventricular (AV) node, establishing a direct link between the atrium and the ventricle (called Kent Bundle). The 12-lead electrocardiogram (ECG) is characterized by a shortened PR, prolonged QRS, and Delta wave $^{1,2}$. Arrhythmias are frequent in WPW syndrome patients due to paroxysmal supraventricular tachycardia (PSVT), atrial fibrillation (AF), etc.; the most dangerous are ventricular fibrillation and sudden death $^{1,2}$.

Nowadays, radiofrequency catheter ablation (RFCA) of APs requires precise localization of the AP along the mitral and tricuspid annulus (gold standard) ${ }^{2}$. The 12-lead ECG is the first step for localization of AP in patients with WPW syndrome, up to now. The data obtained from the ECG parameters can be helpful in planning and shortening the RFCA procedure and fluoroscopy time ${ }^{2}$.
According to many studies, the ratio of left free wall (the side of the mitral valve circle, except the septal location) is very common among APs. Localizing APs includes the left free wall and includes left anterolateral (LAL), left lateral (LL), and left posterolateral (LPL) pathways that can help quickly locate and approach an AP location- to facilitate mapping and to shorten time of radiofrequency ablation ${ }^{1,3-6}$.

Some published ECG algorithms have been based on predicting locations of left free wall APs ${ }^{1,2,4,5,7}$. However, many studies have shown that it is difficult to compare differences between other locations on the side of the mitral valve circle ${ }^{4,5,7}$. Therefore, the purpose of this study was to analyze the 12-lead ECG for septal AP localization and successful RFCA to develop new ECG algorithms using simple parameters, as well as testing the algorithm to predict AP location.

\section{METHODS}

\section{Study design}

The study was observational, cross-sectional, retrospective and prospective. We selected specificity as the standard for the study. 
Patient inclusion and exclusion criteria

\section{Patient inclusion criteria}

The patients included in the study must have typical WPW syndrome and have a single anterograde AP which was successfully identified by RFCA.

\section{Patient exclusion criteria}

The patients excluded from the study must have had one of the following criteria: not treated with RFCA, not having enough information during their treatment, not agreeing to participate in the study, not having successful RFCA, or having more than one AP.

\section{Study contents}

We studied 129 patients with typical WPW syndrome who had a single AP in the left free wall location (along the free wall side of the mitral valve annulus), from January 2001 to May 2017, at Vietnam National Heart Institute, Bachmai Hospital. There are two phases of the study:

+ Phase 1: 84 patients with typical WPW syndrome who had a single anterograde left free wall AP identified by successful RFCA were enrolled to build a new ECG algorithm for localizing left free wall APs using simple parameters, from January 2001 to June 2016.

+ Phase 2: Next, the algorithm was tested prospectively in 45 patients to compare with the location of left free wall AP successfully ablated by RFCA, from June 2016 to May 2017.

WPW syndrome was defined by the 12-lead ECG, characterized by a shortened PR interval $<120$ milliseconds, prolonged QRS duration $\geq 110$ milliseconds, and with a delta wave. Secondary ST and T wave changes were directed opposite to the major Delta wave and QRS vector ${ }^{1}$. Localization of APs was identified by successfully ablation by RCFA (gold standard $)^{2}$.

The annulus position of left free wall AP has 3 regions: left anterolateral (LAL), left lateral (LL), and left posterolateral (LPL). RFCA for APs is conducted through the ablating action of the catheter tip at the intersection of the AP and mitral valve or tricuspid valve. Localization of APs is confirmed by the catheter position for successful RFCA of each location (gold standard $)^{2}$.

The medical treatment was conducted per the procedure in the catheterization laboratory at Vietnam National Heat Institute, Bachmai General Hospital, Hanoi, Vietnam. We perform a full spectrum of cardiac and vascular interventions using the Phillips Advanced Allura Xper FD20 X-ray Biplane System (Phillips, Holland), a programmed cardiac stimulator instrument (integrated in the cardiac stimulator EP-4 System), Cardiac Stimulator EP-4 System, Radio Ampere Generator instrument, diagnostic EP catheter, and mapping and ablation catheter (St. Jude, USA).

\section{Statistical Analysis}

We set the AP location (confirmed by successful RFCA) as the dependent variable and the 12-lead ECG parameters as the independent variables. Pvalues $<0.05$ were considered statistically significant. We calculated values of sensitivity (Se), specificity (Sp), positive predictive value (PPV), and negative predictive value (NPV) for the predicted AP location. The SPSS v. 21.0 software (IBM, USA) was used for analyzing the data.

\section{Research ethics}

The research proposal, database, medical records, and results were approved by the Committee of Science of Vietnam University of Military Medicine. The RFCA used in the research has been demonstrated to be highly effective and safe. The indications and safety of the medical treatment were approved by the Ethics Committee of Bachmai Hospital. The patients were explained about the procedure and provided signed consent for catheter ablation. All patients' medical records were kept confidential and used for research purposes only.

\section{RESULTS}

The study population consisted of 129 patients: 71 men (55\%) and 58 women (45\%), with age of $45.1 \pm$ 14.6 years (range from 19 to 79 years of age).

\section{Characteristics of 12-lead ECG for localiza- tion of septal AP}

The study population consisted of 84 patients (Group I): 40 men (52.4\%) and 40 women (47.6\%), with age of $44.9 \pm 14.5$ years (range from 19 to 79 years of age) Left free wall APs were found in 84 patients: 55 patients had left lateral (65.5\%), 17 patients had left anterolateral (20.2\%), and 12 patients had left posterolateral $(14.3 \%)$.

\section{Transition characteristics of the QRS complex on 12-lead ECG with left free wall location}

Classified transition of left free wall location was most common at after V1,V2 lead (V3-V6) or before V1 lead; this was found in 72 patients (i.e. $85.7 \%$ of the total number of 84 left free wall pathways). 


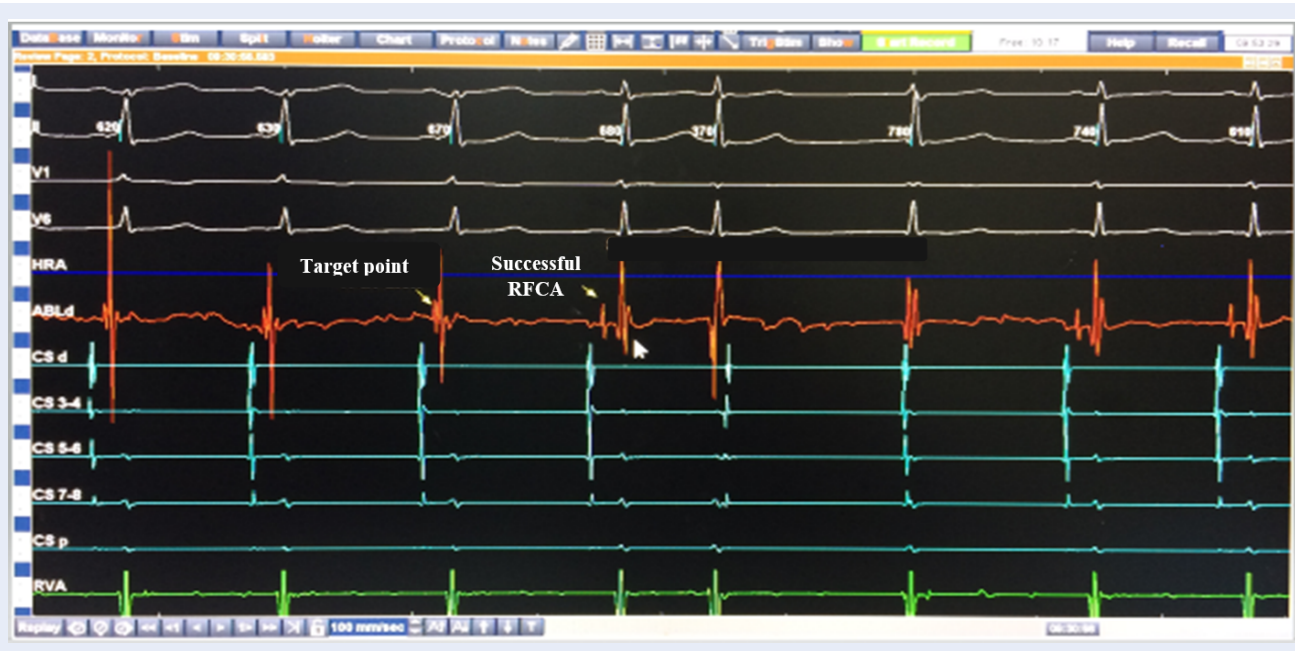

Figure 1: ECG before ablation (Target point: Target for ablation - AP potential with atrial and ventricular wave were mixed) and successful RFCA of AP (atrial and ventricular wave were separated) at the left anterolateral site (arrow).

Characterization of delta wave polarity in V1 lead with left free wall lateral location

Left free wall pathways had positive delta wave that was most common at V1 lead; this was found in $83 / 84$ patients $(98.9 \%)$.

\section{Characterization of delta wave polarity in at least 2/3 inferior lead with left anterolateral or left posterolateral free wall pathway}

Left anterolateral pathways had positive delta wave that were most common in at least $2 / 3$ inferior lead (DII, DIII, AVF); this was found in 17/17 patients (100\%). Meanwhile, left posterolateral pathways had negative delta waves that were most common in at least $2 / 3$ inferior leads; this was found in $11 / 12$ patients $(91.7 \%)$. However, left lateral pathways had positive delta waves that were most common in at least $2 / 3$ inferior leads; this was found in $52 / 55$ patients (94.5\%).

Therefore, the group with "left anterolateral and left lateral pathways" had positive delta waves that were most common in at least $2 / 3$ inferior lead found in $69 / 72$ patients $(95.8 \%)$, and left posterolateral pathways had negative delta wave most common in at least $2 / 3$ inferior lead as above (91.7\%).

For classified R/S ratio of V1 lead for the left anterolateral and left lateral pathway, the anterolateral was most common with $\mathrm{R} / \mathrm{S}>1$ at V1 lead; this was found in 13 patients contributing to $76.5 \%$ among a total number of 17 patients. The left lateral was most common with R/S $<1(62.3 \%)$ or QRS complex'R mor- phology at V1 lead; this was found in 41 patients contributing to $74.5 \%$ among a total number of 55 patients with left lateral pathways. Moreover, left lateral had R/S > 1 at V1 lead in 14/55 patients (25.5\%) Table 1 .

\section{Accuracy of the new ECG algorithm for lo- calizing APs}

The study population consisted of 45 patients (Group II): 27 men (60\%) and 18 women (40\%), with age of $45.6 \pm 15.0$ years (range from 19 to 70 years of age).

Left free wall pathways were found in 45 patients, with $25 / 45$ patients having left lateral (54.3\%), 12/45 patients having left anterolateral $(26.7 \%)$, and $8 / 45$ patients having left posterolateral (17.8\%) pathways.

\section{Localization of free wall lateral AP by tran-} sition characteristics of the QRS complex on 12-lead ECG

The accuracy of the algorithm for localizing APs in left free wall location by QRS complex transition was most common at/after V1,V2 lead (V3-V6) or before V1 lead; this was found in 44 patients, contributing to $97.8 \%$ among the total number of 45 left free wall pathways (sensitivity was $97.8 \%$ ).

\section{Localization of left AP pathways by delta wave polarity in $\mathrm{V} 1$ lead}

The accuracy of the algorithm for localizing APs in free wall pathways belongs to the left side location by positive delta wave, which was most common at the 
Table 1: Characterization of delta wave at $\mathrm{V} 1$ lead, delta way polarity in at least $2 / 3$ inferior leads and R/S ratio at $\mathrm{V} 1$ lead for the left free wall pathways

\begin{tabular}{lccc}
\hline \multicolumn{1}{c}{$\begin{array}{c}\text { Characterization } \\
\text { Left free wall }\end{array}$} & $\begin{array}{c}(+/) \text { Delta in } \\
\text { V1 lead }\end{array}$ & $\begin{array}{c}(+/-) \text { Delta in at least } \\
\mathbf{2 / 3} \text { inferior lead }\end{array}$ & R/S in V1 \\
Left anterolateral & $(+)$ & $(+)$ & $\mathrm{R} / \mathrm{S}>1$ \\
Left lateral & $(+)$ & $(+)$ & $\mathrm{R} / \mathrm{S}<1$ or QRS: R \\
Left posterolateral & $(+)$ & $(-)$ & \\
\hline
\end{tabular}

V1 lead; this was found in $44 / 45$ patients (97.8\%), giving a sensitivity of $97.8 \%$.

Localization of other positions in the left free wall APs by positive or negative delta waves in at least 2/3 inferior leads

The accuracy of the algorithm for localizing APs in left anterolateral and left lateral by positive delta wave in at least $2 / 3$ inferior lead (DII, DIII, AVF) were 12/12 (100\%) and 25/25 (100\%), respectively. Therefore, the accuracy of the algorithm for localizing APs in the group of "left anterolateral and left lateral pathway" or "left posterolateral pathway", by positive delta wave or negative delta waves, in at least 2/3 inferior lead was very significantly high, giving a sensitivity of $100 \%$, specificity of $75 \%$, PPV of $94.9 \%$ and NPV of $100 \%$.

Localization of left anterolateral or left lateral pathways by the $R / S$ ratio at $V 1$ lead

The accuracy of the algorithm for localizing APs was evaluated in the left anterolateral or left lateral position by R/S ratio > $1(91.7 \%)$ or R/S ratio < $1(40 \%)$, and by QRS complex morphology's R pattern (36\%) at V1 lead. Therefore, the accuracy of the two position was significantly higher, giving a sensitivity of $91.7 \%$, specificity of $76 \%$, PPV of $64.7 \%$, and NPV of $95 \%$. Overall, the accuracy of the new ECG algorithm for localizing APs sites showed high accuracy (Tables 2, 3 and 4$)$.

Patients $(n=45)$ with left free wall AP required less procedure time (ablation time was $42.4 \pm 12.8 \mathrm{~min}$ ) and less fluoroscopy time $(7.2 \pm 3.4 \mathrm{~min})$.

\section{DISCUSSION}

\section{Characterization}

Transition characteristics of the QRS complex on 12-lead ECG with left free wall location

Classified transition of left free wall location was most common at before V1 lead (Figure 4A,C,D) or after V1V2 lead (Figure 4B), as noted (85.7\%). This is a characteristic that can be different between free wall and septal location. Some studies have shown that transition of QRS complex can be used for predicting locations of septal or free wall AP but these studies have only focused on differences between right free wall and right septal location. Most studies have not yet found differences between left free wall and left septal location ${ }^{1,3,4}$. Thus, we can predict that the left free wall belongs to free wall location by QRS transition at before V1 or after V1V2 lead.

\section{Characterization of delta wave polarity in V1 lead with left free wall}

Left free wall had positive delta wave that was most common at V1 lead (100\%) (Figure 4). This is very useful in selecting the approach of the catheter- vein or artery. Left-side AP were ablated with retrograde arterial approach (while right-sided AP were ablated with the use of transvenous atrial approach through the femoral vein) ${ }^{2}$.

Some ECG algorithms have been published that can predict locations of left-sided or right-sided APs by positive/negative delta wave ${ }^{1,2}$. Moreover, other studies showed the diagnosis of left or right-sided APs by other ECG parameters; D’Avila A. et al. (1995) showed that positive or negative QRS complex in V1 can be used in diagnosis of left or right-sided $\mathrm{AP}^{3}$. Chiang C.E. et al. (1995) showed that an R/S ratio >1 suggested a left-sided AP ${ }^{4}$. Arruda M.S. et al. (1998) also found that $\mathrm{R} / \mathrm{S}>1$ in $\mathrm{V} 1$ lead corresponded to the left free location and $\mathrm{R} / \mathrm{S}<1$ in $\mathrm{V} 1$ lead corresponded to the right side ${ }^{5}$. Dar M.A. et al. (2008) also used an $\mathrm{R} / \mathrm{S}$ ratio $<1$ in a VL lead to predict left side ${ }^{6}$. Taguchi N. et al. (2014) used R/S ratio $<0.5$ or R/S $>0.5$ in $\mathrm{V} 1$ lead to predict right or left-sided $\mathrm{APs}^{7}$. We had not known that R/S ratio at a VL lead as well as R/S ratio (or QRS) in V1 lead could suggest a left free wall pathway.

Characterization of delta wave polarity in at least 2/3 inferior lead (DII, DIII, AVF) with left anterolateral or left posterolateral pathway

A characteristic electrocardiogram of a patient with left anterolateral pathway showed strongly positive delta waves in at least $2 / 3$ inferior leads (II, III, aVF), 


\begin{tabular}{|c|c|c|c|c|}
\hline $\begin{array}{l}\text { Location } \\
(+/-) \text { Delta wave in } \\
\text { inferior lead }\end{array}$ & $\begin{array}{l}\text { Left antero-lateral } \\
\text { (n) }\end{array}$ & $\begin{array}{l}\text { Left lateral } \\
\text { (n) }\end{array}$ & Left postero- lateral (n) & Total \\
\hline $\begin{array}{l}\text { Positive delta wave in at } \\
\text { least } 2 / 3 \text { inferior }\end{array}$ & 12 & 25 & 2 & 39 \\
\hline $\begin{array}{l}\text { Negative delta wave in at } \\
\text { least } 2 / 3 \text { inferior }\end{array}$ & 0 & 0 & 6 & 6 \\
\hline Total (n) & 12 & 25 & 8 & 45 \\
\hline
\end{tabular}

Table 3: Characterization of R/S ratio at V1 for the left anterolateral and left lateral pathways

\begin{tabular}{|c|c|c|c|c|c|}
\hline \multirow{2}{*}{$\begin{array}{l}\text { Location } \\
\mathrm{R} / \mathrm{S} \text { ratio at } \mathrm{V} 1\end{array}$} & \multicolumn{2}{|c|}{ Left antero lateral } & \multicolumn{2}{|c|}{ Left lateral } & \multirow{2}{*}{ Tota } \\
\hline & & Rate (\%) & & Rate (\%) & \\
\hline $\mathrm{R} / \mathrm{S}>1$ & 11 & $91,7 \%$ & 6 & $24 \%$ & 17 \\
\hline $\mathrm{R} / \mathrm{S}<1$ & 0 & $0 \%$ & 10 & $40 \%$ & 10 \\
\hline $\begin{array}{l}\text { QRS morphology: } \\
\text { R pattern }\end{array}$ & 1 & $8,3 \%$ & 9 & $36 \%$ & 10 \\
\hline Total (n) & 12 & & 25 & & 37 \\
\hline
\end{tabular}

Table 4: Sensitivity, Specificity, PPV and NPV value from the roposed algorithm for the left free wall accessory pathway in 45 patients

\begin{tabular}{lcccc}
\hline \multicolumn{1}{c}{ Left free wall accessory pathways $(\mathbf{n}=\mathbf{4 5})$} & Se (\%) & Sp (\%) & PPV (\%) & NPV (\%) \\
$\begin{array}{l}\text { Sensitivity for accuracy for the free wall lateral } \\
\text { APs }\end{array}$ & $97.8 \%$ & & & \\
Left side APs (left free lateral APs) & $97.8 \%$ & & \\
$\begin{array}{l}\text { Group of "left anterolateral and left lateral" } \\
\text { with "left posterolateral" APs }\end{array}$ & $100 \%$ & $75 \%$ & $94.9 \%$ & $100 \%$ \\
$\begin{array}{l}\text { Left anterolateral or left lateral by R/S ratio at } \\
\text { V1 lead }\end{array}$ & $91.7 \%$ & $76 \%$ & $64.7 \%$ & $95 \%$ \\
\hline
\end{tabular}

as noted (100\%) (Figure 4A), while the left posterolateral pathway group had strongly negative delta waves in at least $2 / 3$ inferior leads (II, III, aVF), as noted (91.7\%) (Figure 4D). Specialized, left lateral pathway had positive delta wave that were most common at least $2 / 3$ inferior leads, a noted (94.5\%) (Figure 4B,C) as the same characteristic left anterolateral pathway (Figure 4A). Therefore, one can build a group that includes "left anterolateral and left lateral pathways", in which the positive delta wave was most common at least in 2/3 inferior lead, as noted (95.8\%), and "left posterolateral pathways" had negative delta waves that were most common at least $2 / 3$ inferior lead, as above (91.7\%).

According to the statistics as above, the left anterolateral and left lateral pathways are often found in the positive delta location in at least $2 / 3$ inferior lead. We evaluated the characteristic R/S ratio at V1 lead in these two regions. The result showed that the left anterolateral position was most common with $\mathrm{R} / \mathrm{S}$ ratio $>1$ at V1 lead (76.5\%), while the left lateral was most common with $\mathrm{R} / \mathrm{S}<1$ (Figure $4 \mathrm{~B}$ ) or QRS complex's $\mathrm{R}$ morphology (Figure 4C) at V1 lead (74.5\%). Thus, these parameters (R/S) help to suggest that left antero lateral or left lateral pathways exist but not at high accuracy. This can be explained by the fact that between two location there are plenty of sequential zones that are similar in electrophysiological angle; thus, there can be many similarities.

Many studies have show the difficulty in localizing between left anterolateral or left lateral pathways, as well as difficulty in discerning differences between other positions of the left free wall pathways. Taguchi N. et al. (2014) selected two locations with "LA \& LL pathways" and "LP \& LPL pathways" ${ }^{7}$. Iturralde T.P. et 
al. (1996) selected two locations for left free wall that included "LPL \& LAS pathways" and "LIP \& LI pathways" ${ }^{8}$.

Thus, we suggest that "LA \& LL pathways" and "LPL pathway" are represented by positive or negative delta wave in at least $2 / 3$ inferior, different between LA and LL position by $\mathrm{R} / \mathrm{S}$ ratio $>1$ or $\mathrm{R} / \mathrm{S}<1$, and $\mathrm{R}$ in $\mathrm{V} 1$ lead (Table 1Figure 3). This can help the doctors to perform a rapid onset of AP location on the mitral valve forwards or backwards, or within $1-2 \mathrm{~cm}$ of the valve, and can help to facilitate mapping techniques and shorten time radiofrequency ablation ${ }^{1,2}$.

\section{Accuracy of new ECG algorithm for localiz- ing left free wall AP}

We have developed a new algorithm using some simple ECG parameters in the left free wall belong to left side pathways, represented by positive delta wave at V1 lead, left free wall site APs by QRS complex transition at before V1 lead or after V1V2 lead. "Left anterolateral and left lateral" group or left posterolateral sites of Aps showed positive/negative delta waves in at least $2 / 3$ inferior; left anterolateral and left lateral had $\mathrm{R} / \mathrm{S}>1$ or $\mathrm{R} / \mathrm{S}<1, \mathrm{R}$.

The algorithm was then tested prospectively in 45 patients and compared with the location of APs in successful ablation by RF. The sensitivity, specificity, positive predictive value and negative predictive value of the diagnosed algorithm for AP sites were then calculated to high accuracy (Table 4).

\section{Localization of free wall lateral location (AP group) by transition characteristics of the QRS complex on 12-lead ECG}

Accuracy of the algorithm for localizing APs in left free wall location by QRS complex transition was at after V1,V2 lead (V3-V6) or before V1 lead, with very high sensitivity of $97.8 \%$.

Accordingly, Dar M.A. et al. (2008) showed that the septal or lateral APs occurred at and by the QRS complex transition at V1V2 or after V1V2 (lead with Se of $97 \%$ and $\mathrm{Sp}$ of $95 \%)^{6}$.

\section{Localization of left AP pathways by delta wave polarity in $\mathrm{V} 1$ lead}

The accuracy of the algorithm for localizing APs in left free wall pathways belong to left location by positive delta wave most common at V1 lead with very high accuracy. Given a sensitivity of $97.8 \%$, Chang C.E. et al. (1995) showed the left side or right side pathways by positive/negative delta wave at V1 lead with Se of $94.4 \%$ and $\mathrm{Sp}$ of $87.5 \%{ }^{4}$.
Localization of other position of left free wall pathways

Localization of other positions of left free wall pathways by positive delta wave or negative delta wave in at least $2 / 3$ inferior lead

Accuracy of the algorithm for localizing APs in group of "includes left anterolateral and left lateral pathways" or "left posterolateral pathways" by positive/negative delta wave in at least $2 / 3$ inferior lead was very significantly higher, giving a sensitivity of $100 \%$, specificity of $75 \%$, PPV of $94.9 \%$ and NPV of 100\% (Table 2). Dar M.A. et al. (2008) showed that the antero or postero APs by positive/negative delta waves in common at inferior lead had Se and Sp been from $85-100 \%{ }^{6}$.

Localization of left anterolateral or left lateral pathways by the $R / S$ ratio at V1 lead

Accuracy of the algorithm for localizing APs in the left anterolateral or left lateral position by $\mathrm{R} / \mathrm{S}$ ratio > 1 or R/S $<1$, R. However, R/S has been significantly high, giving a sensitivity of $91.7 \%$, specificity of $76 \%$, PPV of $64.7 \%$ and NPV of $95 \%$ (Table 3). However, the predictive value was not high (64.7\%); Therefore, the doctor must be attention to consideration of these cases during procedure of the mapping and radiofrequency ablation.

The potential to predict a successful ablation site can help decrease fluoroscopy time and significantly shorten procedure time (Table 5). By fluoroscopy safety guidelines, according to Singer (1993), mean fluoroscopy time per case $>48 \mathrm{~min}$, and $>4$ cases per week, can cause chromosomal aberration ${ }^{12}$.

\section{CONCLUSIONS}

From our study in 129 patients, we have developed a new algorithm for localizing AP for the left free wall using 84 patients with typical WPW syndrome, and validated it. We found that the left free wall had positive delta wave that was most common at V1 lead (98.9\%), and QRS complex transition was most common at after V1V2 lead or before V1 lead (85.7\%). Anterolateral as well as left lateral pathways had positive delta waves that were most common in at least $2 / 3$ inferior lead (100\%) and posterolateral pathways had negative delta wave that was most common in at least $2 / 3$ inferior lead (91.7\%); they were different in LAL and $\mathrm{LL}$ region by $\mathrm{R} / \mathrm{S}>1$ and $\mathrm{R} / \mathrm{S}<1$ or $\mathrm{R}$.

This algorithm was tested prospectively in 45 patients and compared with the location of APs determined by successful ablation by RFCA. The new algorithm was 


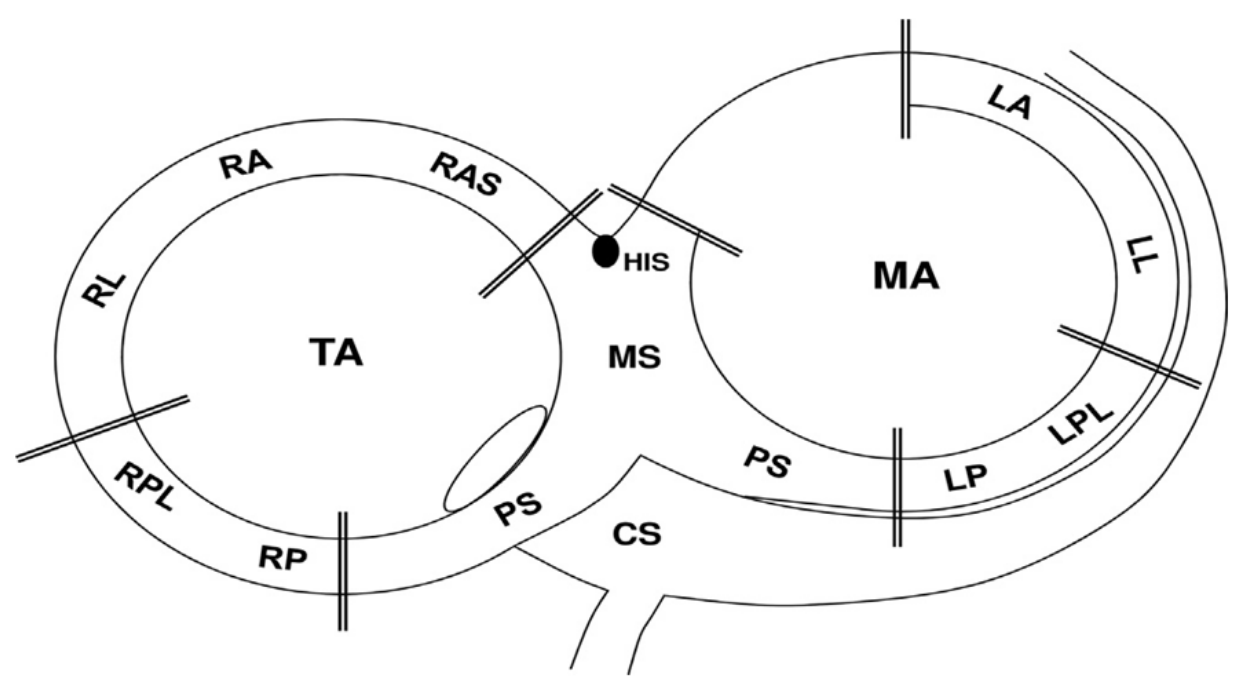

Figure 2: Anatomic definition of AP location. A schematic diagram of the heart (from the left anterior oblique projection) shows the relation among the tricuspid annulus (TA), mitral annulus (MA), His bundle (HIS), coronary sinus (CS), and the anatomic locations of the AP. AP locationsare divided into five main regions, which are defined by double lines. Abbreviations: LA: left anterior; LL: left lateral; LP: left posterior; LPL: left posterolateral; MS: midseptal; PS: posteroseptal; RA: right anterior; RAS: right anteroseptal; RL: right lateral; RP: right posterior; and RPL: right posterolateral (Taguchi N., et al., 2014) ${ }^{7}$.

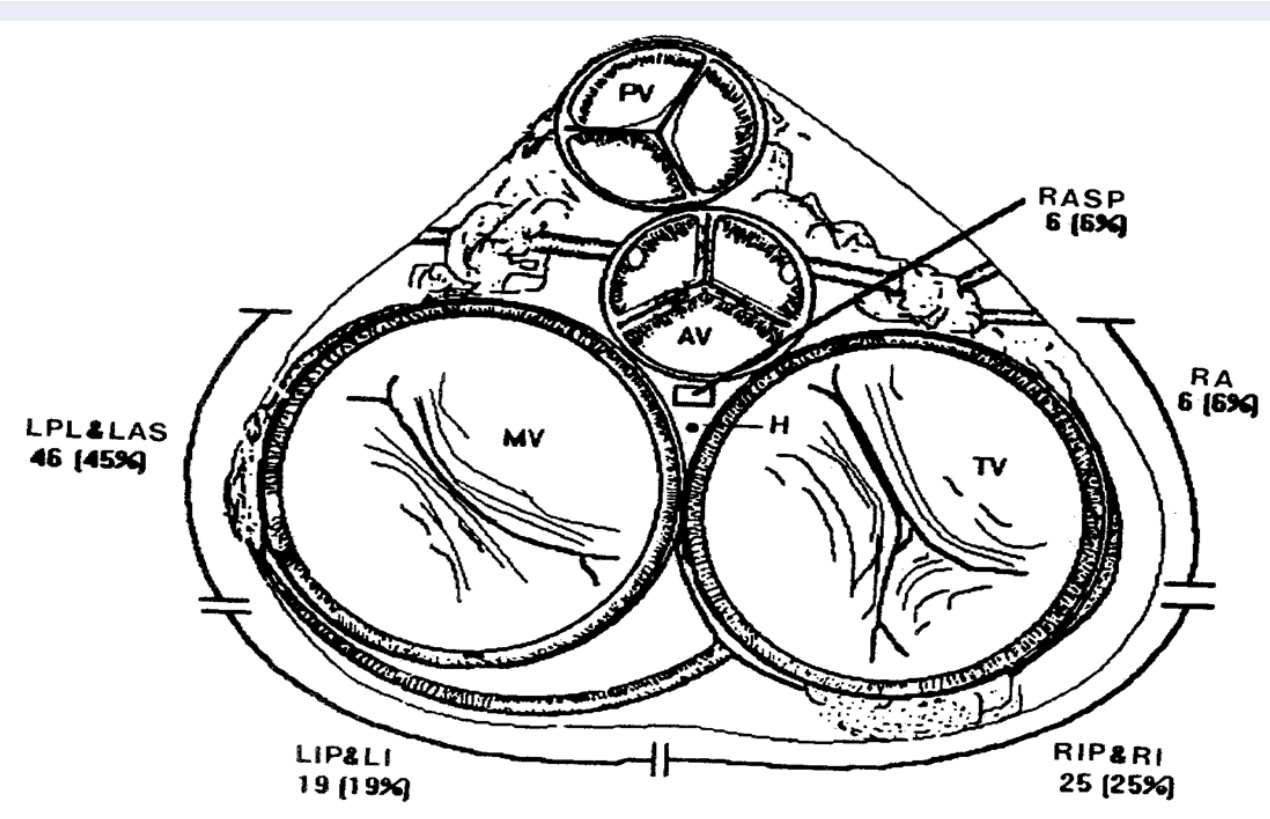

Figure 3: Cross-sectional diagram of the heart at the level of the atrioventricular ring, showing the AP locations in 102 patients undergoing successful radiofrequency catheter ablation of a single manifest AP. Abbreviations: AV: aortic valve; H: bundle of His; LAS, LPL, LI, and LIP: left antero superior, left posterolateral, left inferior, and left inferior paraseptal, respectively; MV: mitral valve; PV: pulmonary valve; RASP, RA, RI, and RIP: right anterosuperior paraseptal, right anterior, right inferior, and right inferior paraseptal, respectively; TV: tricuspid valve (Iturralde T.P., et al.,1996) ${ }^{8}$. 

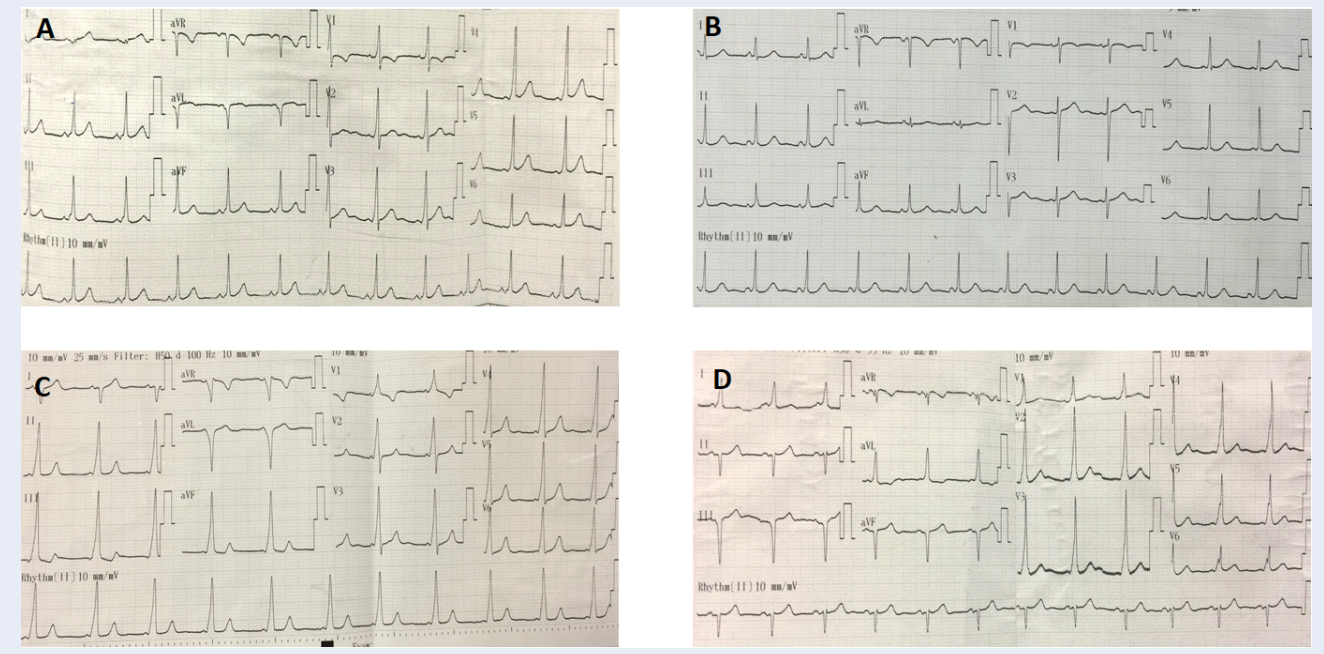

Figure 4: ECG Showing other positions of the left free wall accessory pathways. A. Left anterolateral (Positive delta wave in $\mathrm{V} 1, \mathrm{R} / \mathrm{S}>1$ in $\mathrm{V} 1$, QRS transition at before $\mathrm{V} 1$, and Positive delta wave in $3 / 3$ inferior lead (DII, DIII, aVF)); B. Left Lateral (Positive delta wave in V1, R/S ratio $<1$ in V1, QRS transition at V3, and Positive delta wave in 3/3 inferior lead (DII, DIII, aVF)); C. Left lateral (Positive delta wave in V1, R/S ratio, R morphology in V1, QRS transition at before V1, and Positive delta wave in $3 / 3$ inferior lead (DII, DIII, aVF)); D. Left posterolateral (Positive delta wave in V1, QRS transition at before V1, and Negative delta wave in 3/3 inferior lead (DII, DIII, aVF)).

Table 5: Comparision of proceduralparameters in other studies on procedure time and fluoroscopy

\begin{tabular}{lccc}
\hline \multicolumn{1}{c}{ Authors } & $\begin{array}{c}\text { Accessory athway } \\
\text { Location }\end{array}$ & $\begin{array}{c}\text { Mean Procedure } \\
\text { Time (minute) }\end{array}$ & $\begin{array}{c}\text { Mean Fluoroscopy } \\
\text { Time (minute) }\end{array}$ \\
Lemery R. et al. $(1992)^{9}$ & Left free wall $(\mathrm{n}=36)$ & $162.0 \pm 48.0$ & $46.0 \pm 24.0$ \\
Dong T.V. (2006) ${ }^{10}$ & Left lateral AP ( $\mathrm{n}=47)$ & $82.9 \pm 33.0$ & $18.5 \pm 10.7$ \\
& Left posterolateral ( $\mathrm{n}=27)$ & $82.4 \pm 51.6$ & $19.9 \pm 13.2$ \\
& Left anterolateral $(\mathrm{n}=21)$ & $92.8 \pm 42.7$ & $23.3 \pm 9.5$ \\
Schwagten B. et al. $(2010){ }^{11}$ & Left side AP $(\mathrm{n}=10)$ & $87.1 \pm 30.8$ & $14.4 \pm 4.7$ \\
Our research (2016-2017) & Left free wall $(\mathrm{n}=45)$ & $42.4 \pm 12.8$ & $7.2 \pm 3.4$ \\
\hline
\end{tabular}

demonstrated to have high accuracy as well as sensitivity (from $75 \%$ to $100 \%$ ), and could facilitate radiofrequency ablation in patients with left side AP as well as left free wall pathways. The potential to predict AP location is very important in affecting fluoroscopy time and procedure time (by significantly shortening them), when using the parameters as above for the prediction criteria of the left free wall location.

\section{COMPETING INTERESTS}

The authors declare that there is no conflict of interest regarding the publication of this article.

\section{AUTHORS' CONTRIBUTIONS}

Si CD: designed the study, collected data, conducted the statistical analysis and wrote the manuscript.
Khanh PQ and Dong TV: are revised the manuscript during the review process.

\section{ACKNOWLEDGEMENTS}

Vietnam University of Military Medicine \& Vietnam National Heart Institute, Bachmai Hospital.

\section{ABBREVIATIONS}

AP: Accessory pathway

APs: Accessory pathways

AV node: Atrioventricular node

AV: Atrioventricular

ECG: Electrocardiogram

RFCA: Radiofrequency catheter ablation

WPW: Wolff-Parkinson-White 


\section{REFERENCES}

1. Surawicz B, Knilans TK, Chou TC. Chou's Electrocardiography in clinical practice: Adult and Pediatric; 2008.

2. Basiouny T. Prospective Validation of A Sezer ECG Algorithm For Localization of Accrssory Pathways in Patients With WolffParkinson-White Syndrome. Al-Azhar Assiut Medical Journal. 2012;10:127-53.

3. d'Avila A, Brugada J, Skeberis V, Andries E, Sosa E, Brugada P. A fast and reliable algorithm to localize accessory pathways based on the polarity of the QRS complex on the surface ECG during sinus rhythm. Pacing and Clinical Electrophysiology. 1995;18:1615-27. Available from: DOI:10.1111/j.1540-8159. 1995.tb06983.x.

4. Chiang CE, Chen SA, Teo WS, Tsai DS, Wu TJ, Cheng CC, et al. An accurate stepwise electrocardiographic algorithm for localization of accessory pathways in patients with WolffParkinson-White syndrome from a comprehensive analysis of delta waves and $\mathrm{R} / \mathrm{S}$ ratio during sinus rhythm. The American journal of cardiology. 1995;76:40-46.

5. Arruda MS, McClelland JH, Wang X, Beckman KJ, Widman LE, Gonzalez MD. Development and validation of an ECG algorithm for identifying accessory pathway ablation site in WolffParkinson-White syndrome. Journal of Cardiovascular Electrophysiology. 1998;9:2-12. Available from: DOI:10.1111/j.15408167.1998.tb00861.x.

6. Dar MA, SHEIKH SH, ABID AR, MALLICK NH. Localization of ACcessory Pathways According to AP Fitzpatrick Ecg Criteria in Patents with Wolff-Parkinson-White Syndrome in Our Popula- tion. Pakistan Heart Journal. 2012;41.

7. Taguchi N, Yoshida N, Inden Y, Yamamoto T, Miyata S, Fujita M. A simple algorithm for localizing accessory pathways in patients with Wolff-Parkinson-White syndrome using only the R/S ratio. Journal of Arrhythmia. 2014;30:439-43. Available from: DOI:10.1016/j.joa.2013.10.006.

8. Iturralde P, Araya-Gomez V, Colin L, Kershenovich S, de Micheli A, Gonzalez-Hermosillo JA. A new ECG algorithm for the localization of accessory pathways using only the polarity of the QRS complex. Journal of Electrocardiology. 1996;29:289-99. Available from: Doi:10.1016/s0022-0736(96)80093-8.

9. Lemery R, Talajic M, Roy D, Coutu B, Lavoie L, Lavallée E. Suc cess, safety, and late electrophysiological outcome of lowenergy direct-current ablation in patients with the WolffParkinson-White syndrome. Circulation. 1992;85:957-62. Available from: Doi:10.1161/01.cir.85.3.957.

10. Dong TV. Doctor of Philosophy Thesis in Medicine: Research in Electrophysiology and treatment of Wolff-Parkinson-White Syndrome by Radiofrequency Catheter Ablation; 2006.

11. Schwagten $B$, Jordaens $L$, Rivero-Ayerza $M$, Belle $Y V$, Knops $P$ Thornton IA. A randomized comparison of transseptal and transaortic approaches for magnetically guided ablation of left-sided accessory pathways. Pacing and Clinical Electrophysiology. 2010;33:1298-303. Available from: DOI:10.1111/ j.1540-8159.2010.02810.x.

12. Singger I. Clinical manual of electrophysiology. In: Clinical manual of electrophysiology. Baltimore: Williams \& Wikins; 1993. p. $52-68$. 